\title{
CONFÉRENCE
}

\section{Gestion des traumatismes oro-maxillo-faciaux et applications spécifiques aux attentats Niçois.}

\section{Dr Savoldelli C}

IUFC, CHU Nice

La chirurgie des traumatismes faciaux est la discipline visant à traiter les traumatismes du crâne et de la face ainsi que leurs séquelles. Le degré de complexité de la prise en charge est très variable selon la sévérité des dégâts causés par le traumatisme. Les lésions peuvent aller d'une simple fracture des os du nez ou la mandibule (mâchoire inférieure) au fracas faciaux associant de multiples fractures de la face et des lésions des tissus mous.

La particularité de la prise en charge de ces traumatismes est d'offrir un traitement aussi bien fonctionnel (rétablissement de l'occlusion ou de l'oculomotricité) qu'esthétique (plaies et déformations). L'évaluation de la gravité des lésions chez un patient présentant un traumatisme facial nécessite un diagnostic lésionnel initial complet. La connaissance de l'anatomie et la pratique d'un examen clinique systématisé permettent ce diagnostic, qui sera confirmé par la réalisation raisonnée d'imagerie. Nous rappellerons les bases anatomiques du massif facial ainsi que l'examen clinique et paraclinique du traumatisé facial. Nous développerons plus en détails la prise en charge de ces traumatismes et l'aspect singulier des traumatismes faciaux causés par l'attaque terroriste survenue à Nice le 14 juillet 2016.

savoldelli.c@chu-nice.fr

(C) The authors, published by EDP Sciences. This is an Open Access article distributed under the terms of the Creative Commons Attribution License 4.0 (http://creativecommons.org/licenses/by/4.0/). 